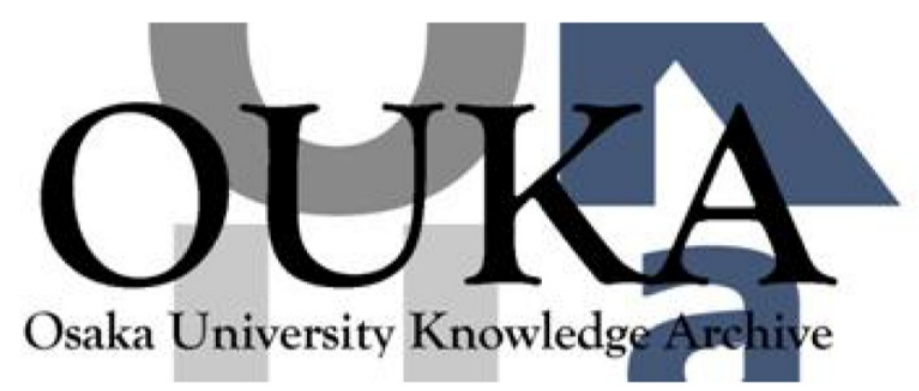

\begin{tabular}{|c|l|}
\hline Title & $\begin{array}{l}\text { Thin observation module by bound optics (TOMBO) } \\
\text { : concept and experimental verification }\end{array}$ \\
\hline Author(s) & $\begin{array}{l}\text { Tanida, Jun; Kumagai, Tomoya; Yamada, Kenji et } \\
\text { al. }\end{array}$ \\
\hline Citation & Applied Optics. 40(11) p. 1806-p. 1813 \\
\hline Issue Date & 2001-04-10 \\
\hline oaire:version VoR \\
\hline URL & https://hdl. handle. net/11094/3086 \\
\hline rights & \\
\hline Note & \\
\hline
\end{tabular}

Osaka University Knowledge Archive : OUKA

https://ir. Library. osaka-u. ac. jp/

Osaka University 


\title{
Thin observation module by bound optics (TOMBO): concept and experimental verification
}

\author{
Jun Tanida, Tomoya Kumagai, Kenji Yamada, Shigehiro Miyatake, Kouichi Ishida, \\ Takashi Morimoto, Noriyuki Kondou, Daisuke Miyazaki, and Yoshiki Ichioka
}

\begin{abstract}
A compact image-capturing system called TOMBO (an acronym for thin observation module by bound optics) is presented in which the compound-eye imaging system is utilized to achieve a thin optical configuration. The captured multiple images are processed to retrieve the image of the target object. For image retrieval, two kinds of processing method are considered: image sampling and backprojection. Computer simulations and preliminary experiments were executed on an evaluation system to verify the principles of the system and to clarify the issues related to its implementation. (C) 2001 Optical Society of America
\end{abstract}

OCIS codes: $110.2970,110.4190,100.3010,130.0250,230.3120$.

\section{Introduction}

Data acquisition is a crucial issue in today's information-oriented society. Acquisition and storage of images are particularly important, and they are key issues for research. In spite of efforts to reduce the size of photographic cameras and video recorders, these devices are still relatively large for easy portability. Therefore, it is desirable to design morecompact equipment based on different principles.

For compact imaging, a system that uses an array of microlenses seems promising. ${ }^{1-3}$ The original idea comes from the multiple-imaging system of arthropods, ${ }^{4,5}$ by emulation of which a wide field of view can be achieved with lightweight and compact imag-

J. Tanida (e-mail: tanida@mls.eng.osaka-u.ac.jp) and T. Kumagai are with the Department of Material and Life Science, Graduate School of Engineering, Osaka University, 2-1 Yamadaoka, Suita 565-0871, Japan. K. Yamada is with the Osaka Science and Technology Center, 2-7-1 Ayumino, Izumi, Osaka 5941157, Japan. S. Miyatake, K. Ishida, and T. Morimoto are with Research and Development Headquarters, Minolta Company, Ltd., 1-2 Sakura-Machi, Takatsuki, Osaka 569-8503, Japan. N. Kondou is with Dainippon Screen Manufacturing Company, Ltd., Teranouchi-agaru 4, Horikawa-dori Kamigyo-ku, Kyoto 602-8585, Japan. D. Miyazaki is with the Osaka City University, 3-3-138 Sugimoto, Sumiyoshi-ku, Osaka 558-8585, Japan. Y. Ichioka is with the Nara National College of Technology, 22 Yata-cho, Korimaya, Nara 639-1080, Japan.

Received 18 August 2000; revised manuscript received 21 November 2000.

0003-6935/01/111806-08\$15.00/0

(C) 2001 Optical Society of America ing hardware. For example, an artificial visual sensor with $16 \times 16$ pixels was constructed with a microlens-and-photodetector array. ${ }^{1}$ Details of the design and an analysis of compound-eye optical sensors were presented by Sanders and Halford. ${ }^{2}$ Image processing by modification of the compound-eye system has also been described. ${ }^{3}$ Regardless of the efforts dedicated to implementing this idea, no practical device capable of capturing high-resolution images has yet been developed to our knowledge because the number of pixels of the captured image is equal to that of the compound eyes.

In this paper we present a compact imagecapturing system that will act as a portable image grabber with acceptable image resolution. The system is called TOMBO 6,7 (which is an acronym for thin observation module by bound optics) after the Japanese name for the dragonfly, which has a compoundeye imaging system. The TOMBO, which is a combination of a multiple-imaging system and postdigital processing, can provide a compact hardware configuration as well as processing flexibility. In Section 2 we briefly explain a compound-eye system. In Section 3 we present the architecture of the TOMBO, and in Section 4 we describe an experimental TOMBO system. In Section 5 we explain two image-retrieval methods and present some experimental results. Based on the results, the issues related to the implementation are discussed.

\section{Compound-Eye Imaging System}

Figure 1 is a schematic diagram of the compound-eye imaging system. ${ }^{4,5}$ This optical system is composed 


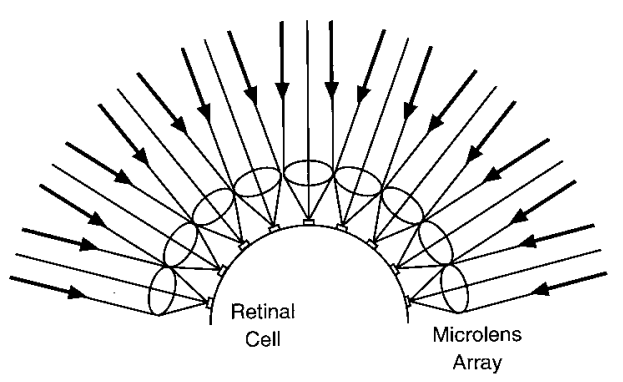

Fig. 1. Compound-eye imaging system.

of multiple sets of elemental optics (called units), each of which consists of a microlens and a photosensitive cell. The object is imaged onto the photosensitive cell by each microlens, and the photo signal at a specific position is sampled and detected by the cell. While adjacent units focus on similar images on the surface, different parts of the object are sampled by the cells owing to the geometrical relationship between the object and the unit. As a result, a set of the signals detected by all the units constitutes the whole image of the object, as shown in Fig. 2. Note that the reconstructed image is an erect one and that its number of pixels is the same as the number of units.

As an interesting characteristic of the compoundeye imaging system is that one can achieve simple image manipulation by changing the position of each photosensitive cell. ${ }^{3}$ For an erect image, the photosensitive cell is set at the optical axis of each unit. In contrast, changing the cell position according to a specific rule enables us to achieve reduction, magnification, or rotation of the object.

The most salient feature of the compound eye's imaging system is its applicability to a wide-field-ofview (as much as 360-deg) optical system with lightweight and compact hardware. ${ }^{2}$ For the single-eye imaging system an extremely large and bulky lens is required, which causes aberrations and weight problems. To compensate for this disadvantage, a moving mechanism is usually equipped with a single-eye imaging system to cover the narrow-field-of-view angle of the imaging system. However, a method to

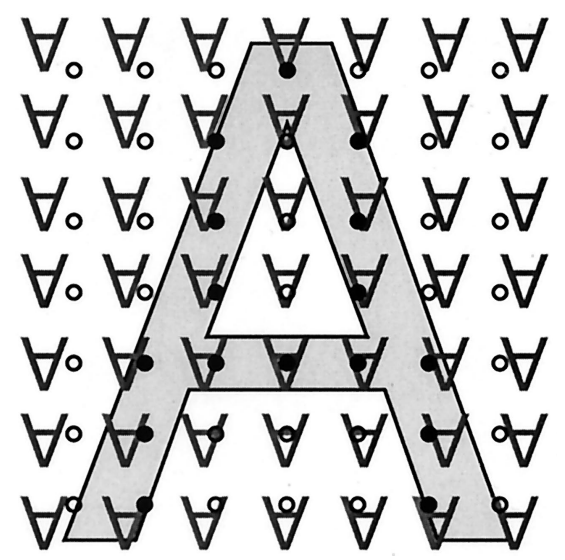

Fig. 2. Erect image retrieved by sampling of multiple images.

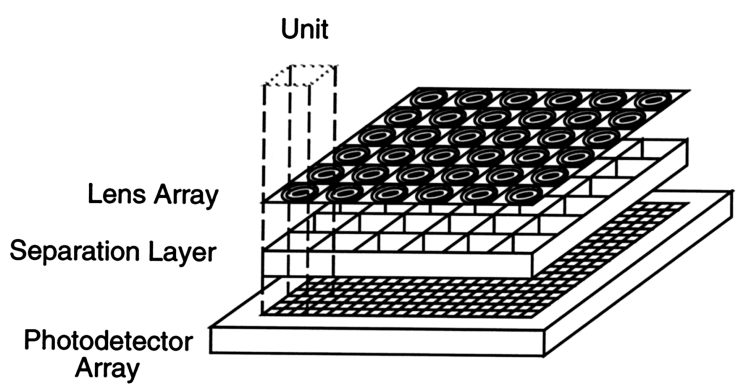

(a)

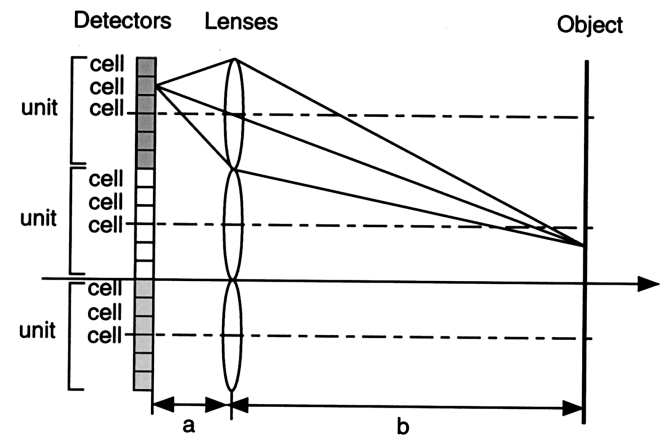

(b)

Fig. 3. TOMBO architecture: (a) system structure and (b) optical system.

control movement is also required and should complicate the imaging system. The compound-eye imaging system is free from this problem and is expected to be useful as a compact image-capturing system.

As an essential problem of the compound-eye imaging system, an insufficient number of units results in degradation of the image quality. In addition, only a part of the incident optical signal is detected by the photosensitive cell, so the light efficiency of the system is relatively low. The TOMBO architecture overcomes these problems to implement a compact image-capturing system.

\section{TOMBO Architecture}

\section{A. Structure and Characteristic Parameters}

Figure 3(a) shows the structure of the TOMBO architecture. ${ }^{6,7}$ The system consists of three components: a microlens array, a separation layer, and a photodetector array. A notable feature of this architecture is that each microlens sends optical signals to the multiple photosensitive cells on the photodetector array and comprises an imaging unit. This structure should be distinguished from the native form of the compound-eye imaging system or a kind of CCD equipped with a microlens array to increase the incident optical flux in which one microlens corresponds to one photosensitive cell. Adjacent units are separated by an opaque wall to prevent cross talk. For the photodetector array, a conventional CCD chip or a complementary metal-oxide semiconductor sen- 
Table 1. Example Combinations of Characteristic Parameters for $\mathbf{N}=$ $739 \times 575$ and $s=11 \mu \mathrm{m} \times 11 \mu \mathrm{m}$

\begin{tabular}{ccc}
\hline$\nu$ & $\mu$ & $d(\mu \mathrm{m})$ \\
\hline $4 \times 4$ & $184 \times 143$ & 44 \\
$8 \times 8$ & $92 \times 71$ & 88 \\
$16 \times 16$ & $46 \times 35$ & 176 \\
$32 \times 32$ & $23 \times 17$ & 352 \\
\hline
\end{tabular}

sor chip can be used. Owing to the small number of components, the structure of the TOMBO is simple, so high productivity is expected.

Figure 3(b) is a side view of the optical system of the TOMBO. The TOMBO is characterized by unit number $\mu$, unit width $d$, and number of photosensitive cells per unit, $v$. For a photodetector array with $N$ pixels and pixel width $s$, the following equations are satisfied:

$$
\begin{aligned}
N & =\mu \nu, \\
s & =d / \nu .
\end{aligned}
$$

The proportion of characteristic parameters $\mu$ and $\nu$ is arbitrary. Table 1 lists examples of the combination for the CCD camera $(N=739 \times 575, s=11$ $\mu \mathrm{m} \times 11 \mu \mathrm{m})$ used in the experimental TOMBO system. Note that the native compound-eye imaging system corresponds to $\nu=1$ and $\mu=N$.

\section{B. Signal Separation by a Wall}

Optical signal cross talk between adjacent units is a troublesome problem in image detection. In a conventional imaging system, an aperture stop is effective in preventing cross talk. However, this solution requires the use of a thick configuration, which is not suitable for the TOMBO architecture. In another effective method, a separation layer is inserted between the microlens and the photodetector arrays. Although a full-height wall that touches both arrays is ideal, a partial wall is still effective in reducing cross talk. As shown in Fig. 4(a), width $x$ of the maximum area affected by the cross talk is determined as follows,

$$
x=\frac{(a-c) d}{2 c},
$$

where the distance between the microlens and the photodetector arrays, the unit width, and the wall height are $a, d$, and $c$, respectively. Note that the separation layer can serve as a structural frame of the system.

\section{Signal Separation by Polarizers}

Two polarizers also provide signal separation. As shown in Fig. 5, a mosaic of small polarizers with orthogonal orientations can be used to prevent the signals of adjacent units from being mixed. Two of the mosaic polarizers are set at the microlens and the photodetector arrays. Then the signal cross talk can be eliminated for the four neighboring units. In this

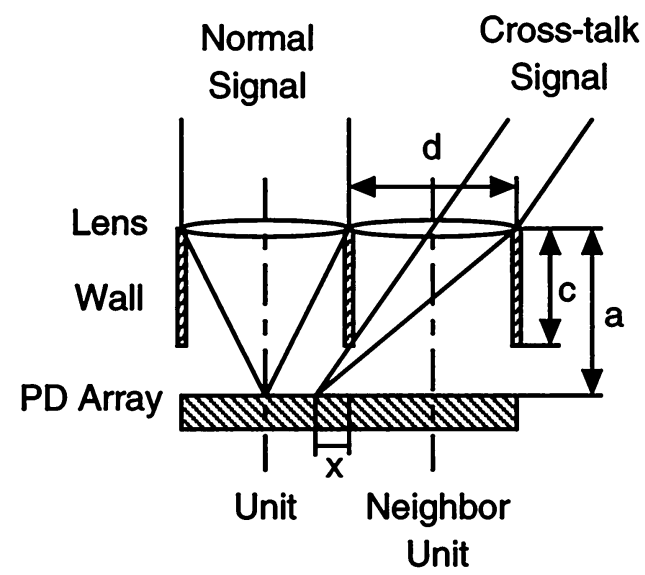

(a)

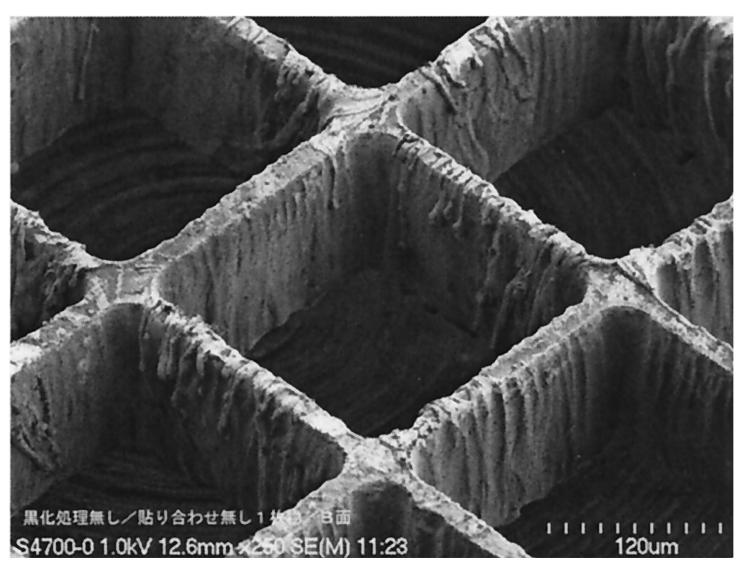

(b)

Fig. 4. Signal-separation layer: (a) optical system and (b) scanning electron microscope picture of a fabricated separation layer. $\mathrm{PD}$, photodiode.

case, each unit detects either of the orthogonal polarizations. As a result, this configuration can be utilized for polarization-sensitive imaging.

\section{Extension of the Field of View}

When the object is located a close distance from the system, the field of view of the system is limited, as shown in Fig. 6(a). To extend the field of view, an array of deflective elements, e.g., a prismlet array, can be utilized, as shown in Fig. 6(b). A concave lens in front of the lens array could serve the same function with a penalty of increasing the system's thickness. A practical way to extend the field of view is to use a diffractive lens accompanied by the beam steering effect. In this case the system's thickness will not be increased.

When the object is located at infinity, all units in the TOMBO observe the identical image, which results in degradation of the observed image. For solution of this problem, the above method is also effective. 


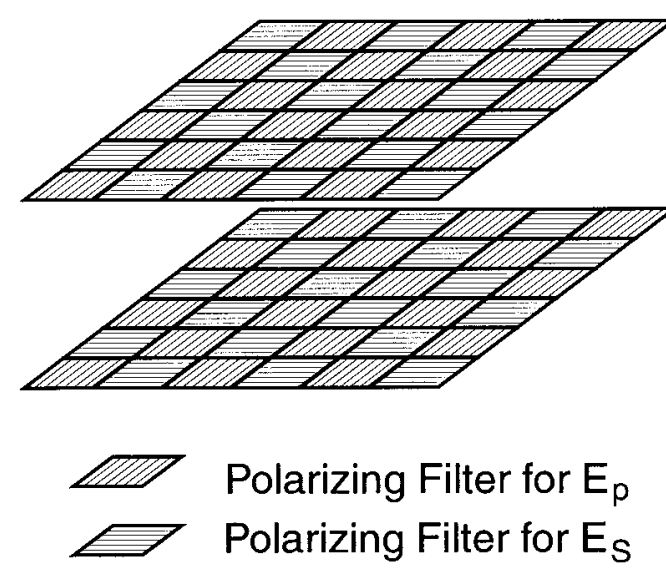

Fig. 5. Polarizing filters for elimination of cross talk.

\section{Experimental TOMBO System}

To evaluate the performance of the TOMBO architecture, we constructed an experimental system consisting of a CCD camera and a planar microlens array. To avoid interference between the microlens and the bonding wires of the CCD chip in the camera, we use a CCD camera (Hamamatsu Photonics, C5948-01; number of pixels, $N=739 \times 575$; cell size, $s=11$ $\mu \mathrm{m} \times 11 \mu \mathrm{m})$ with an imaging fiber plate placed in front of the CCD chip. Therefore we can set a small backfocus, $a$, in the system alignment. For precise positioning, the CCD camera is attached to a holder that is capable of six-axis adjustment, whereas the microlens array is fixed on the base plate. Figure 7 is a photograph of the experimental TOMBO system.

A refractive planar microlens (focal length, 650 $\mu \mathrm{m}$; lens diameter, $250 \mu \mathrm{m}$; Nippon Sheet Glass) is used as a microlens array. In this case, $d=250 \mu \mathrm{m}$ and the characteristic parameters $\mu$ and $\nu$ are $32 \times$
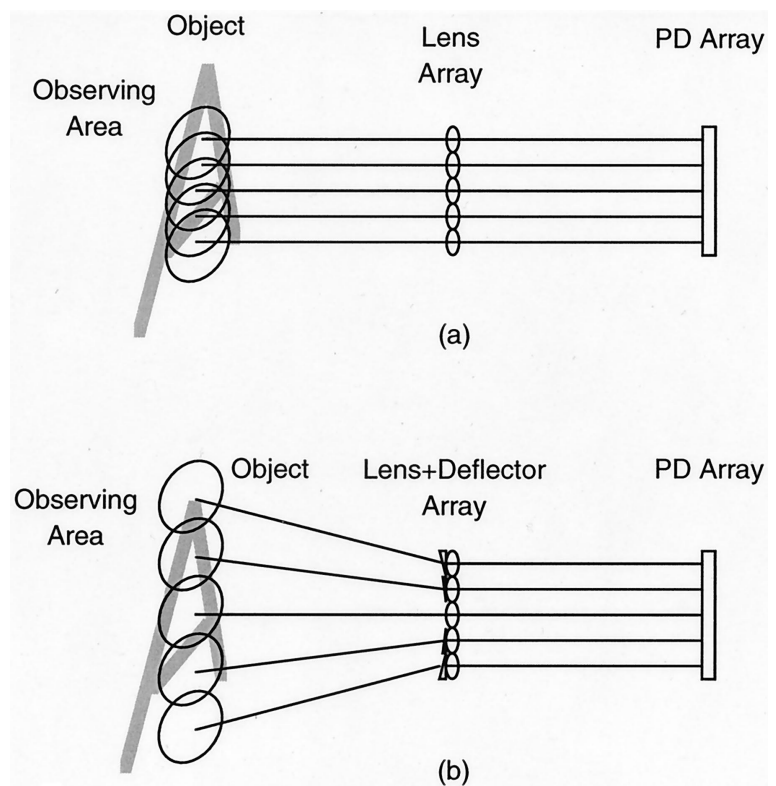

Fig. 6. Field of view of the TOMBO: (a) normal form and (b) wide-view configuration with deflective elements.

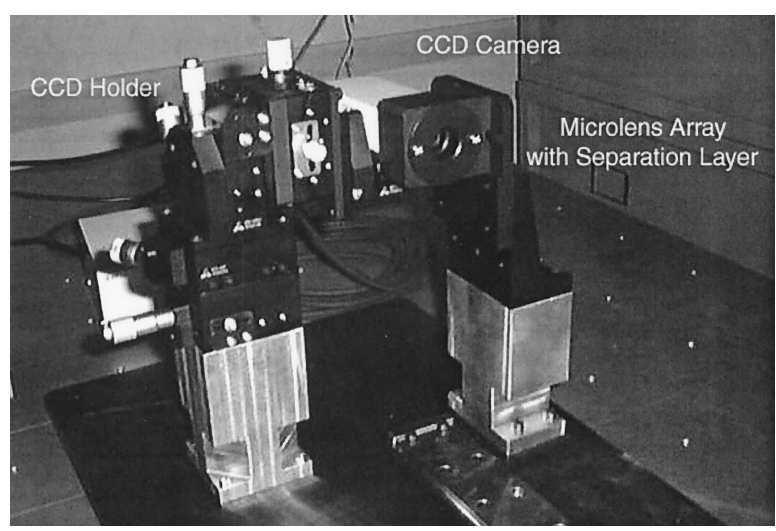

Fig. 7. Photograph of the experimental TOMBO system.

25 and $22.7 \times 22.7$, respectively. Although $v$ is a decimal fraction, this effect can be compensated for if the relation between the positions of the object and the photosensitive cell is included in the retrieve processing.

To avoid signal cross talk between the adjacent units, we insert two stainless-steel plates, each of which is $120 \mu \mathrm{m}$ thick and has an array of squareshaped holes, between the microlens and the face of the fiber plate of the CCD camera. The holes are shaped by a YAG laser beam (output power, $14 \mathrm{~W}$; beam diameter, $15 \mu \mathrm{m}$ ), in which the thickness of the remaining wall is $\sim 30 \mu \mathrm{m}$. Figure $4(\mathrm{~b})$ is a scanning electron microscope picture of the fabricated separation layer. For easy fabrication, two $120-\mu \mathrm{m}$-thick plates were processed; then they were stacked to function as a separation layer of $240-\mu \mathrm{m}$ height.

Observed images without and with the separation layer are depicted in Fig. 8. Although the brightness is reduced, the image with the separation layer shows better contrast than that without the separation layer.

\section{Image-Retrieval Methods}

To retrieve the image of the object from the signals captured by the multiple units, we study the image sampling and the backprojection methods.

\section{A. Sampling Method}

Based on the mechanism of the compound-eye imaging system, an image comprises a set of the signals sampled at the specific points in the individual units. In the native compound-eye imaging system, the sampling is achieved through a small aperture in the photosensitive cell, whereas the TOMBO architecture executes the same operation just by selecting the signal at the detector element of the photodetector array.

As one can imagine, flexibility in selection of the representative signals increases the functionality of the TOMBO system. For observation of an object located a short distance from the system, the signals at the optical axes of the individual units produce an erect image, as shown in Fig. 2. Changing the sam- 


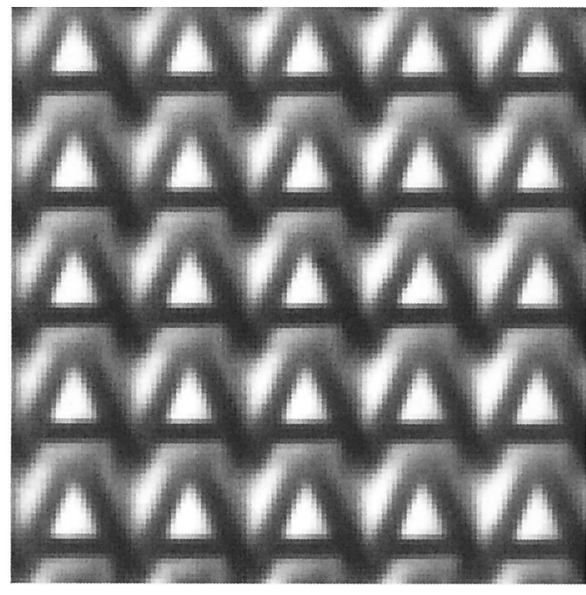

(a)

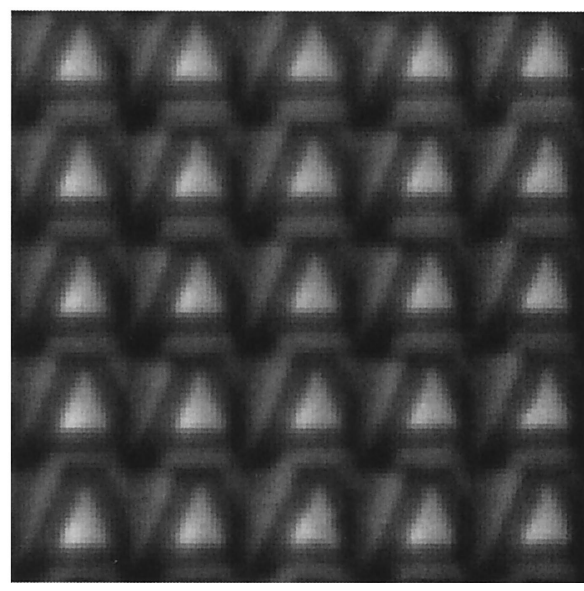

(b)

Fig. 8. Example images captured by the experimental TOMBO system (a) without and (b) with a separation layer.

pling points enables us to transform the image by reduction, magnification, rotation, and so on.

Figure 9 shows example images obtained by the sampling method from the experimental TOMBO system. The object size was $8 \mathrm{~mm} \times 8 \mathrm{~mm}$. The distance between the object and the microlens, $b$, and that between the object and the photodetector, $a$, were $45 \mathrm{~mm}$ and $660 \mu \mathrm{m}$, respectively.

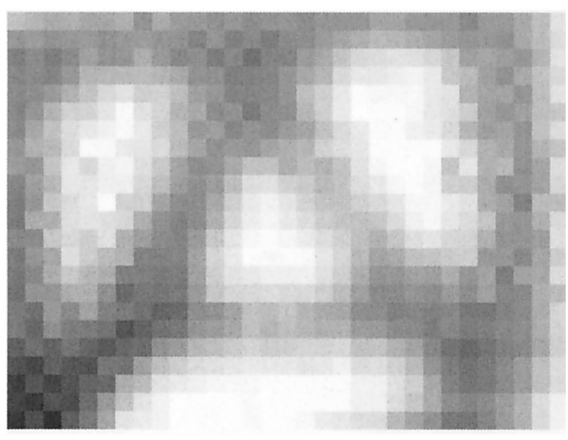

(a)

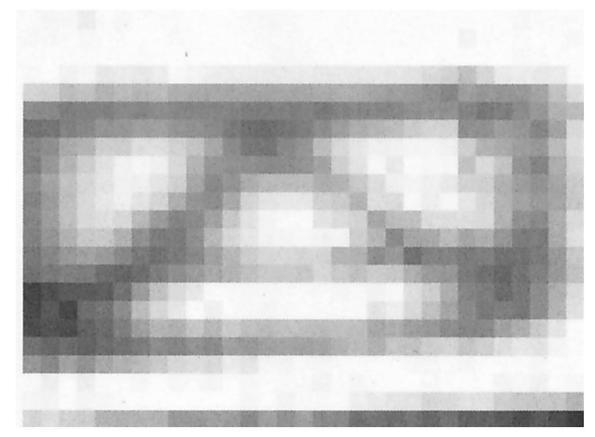

(c)
Although the sampling method is easy to implement, the number of pixels of the retrieved image is determined by unit number $\mu$. Thus, increasing the unit number is crucial for high-resolution imaging. A configuration with small $v$, i.e., a small number of sensors per unit, can relax the fabrication conditions, with the penalty of less functionality.

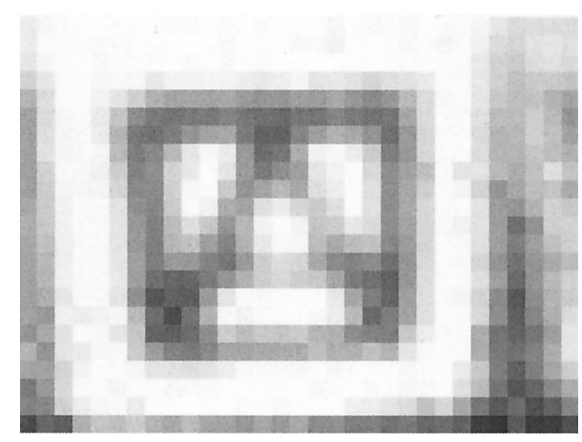

(b)

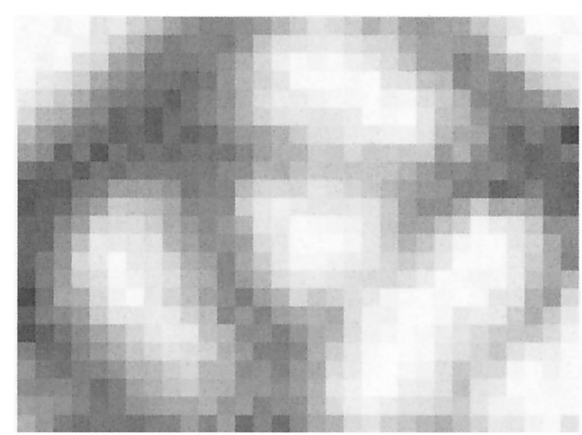

(d)

Fig. 9. Image retrieval by the image-sampling method:

(a) erect, (b) demagnified, (c) stretched, and (d) rotated images. 


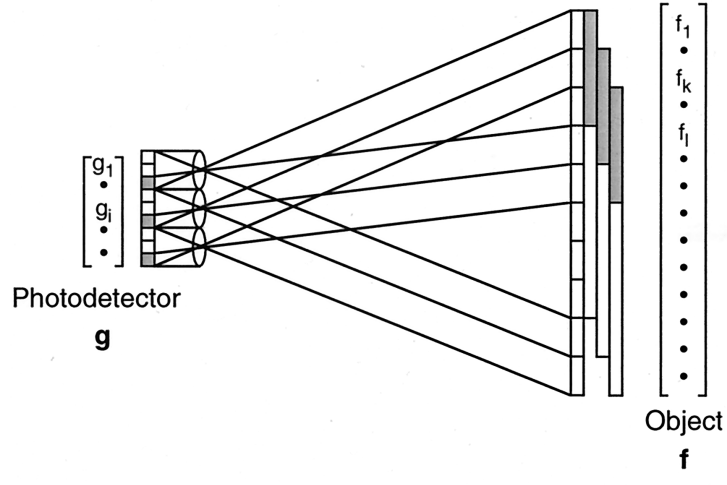

(a)

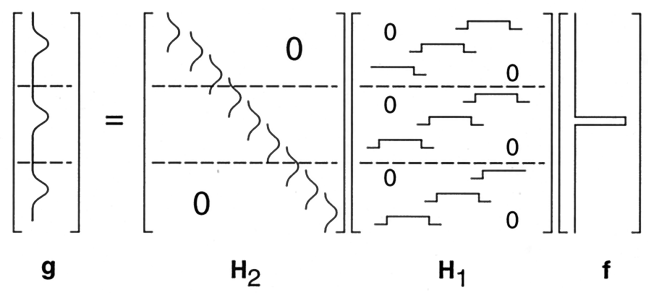

(b)

Fig. 10. Relationship between object and detected signals for $\mu=$ 3 and $v=3$ : (a) geometrical relation and (b) typical form of the system equation.

\section{B. Backprojection Method}

To increase the quality of the reconstructed images, all the signals captured by the photodetector array should be utilized in processing. If we describe the relation between the elements on the object and the photodetectors, we can calculate the object image from the captured signals.

For simplicity, we consider the optical system of a one-dimensional model with vectors $\mathbf{f}$ and $\mathbf{g}$ and matrix $\mathbf{H}$, where $\mathbf{f}$ and $\mathbf{g}$ are the elements of the object and the signals at the photodetector, respectively, and $\mathbf{H}$ denotes the system matrix. Then the TOMBO system is simply described as

$$
\mathbf{g}=\mathbf{H f} \text {. }
$$

Referring to Fig. 10(a) and considering the pointspread function of the optical system of each unit, we can describe the system matrix by the following form,

$$
\mathbf{H}=\mathbf{H}_{2} \mathbf{H}_{1},
$$

where $\mathbf{H}_{1}$ is image duplication with demagnification and $\mathbf{H}_{2}$ is the point-spread function of the imaging units. An example form of Eq. (4) for $\mu=3$ and $v=$ 3 is illustrated in Fig. 10(b). Note that the elements in $\mathbf{H}_{1}$ are easily identified from the system parameters. For $\mathbf{H}_{2}$, relatively complicated calculation is required, with the appropriate assumptions. $\mathbf{H}_{2}$ can also be determined by an experimental measurement with the same condition as the usage.
In general, $\mathbf{H}$ is not necessarily a regular matrix, so one must employ some mathematical techniques to solve Eq. (4). We use the singular-value decomposition method 8,9 to obtain a pseudoinverse matrix $\mathbf{H}^{+}$. In this method, the least-mean-squares criterion is adopted. System matrix $\mathbf{H}$ can be decomposed by use of the singular values as follows,

$$
\mathbf{H}=\mathbf{V W \mathbf { U } ^ { T }},
$$

where $\mathbf{U}$ and $\mathbf{V}$ are matrices composed of the eigenvectors of $\mathbf{H H}^{T}$ and $\mathbf{H}^{T} \mathbf{H}$, respectively. The superscript $T$ means a transpose operator. $\mathbf{W}$ is a singular matrix that has the eigenvalues $w_{i}\left(w_{1}>\right.$ $w_{2}>\cdots>w_{r}$ ) as the diagonal components:

$$
\mathbf{W}=\left[\begin{array}{ccccc}
w_{1} & & & \\
w_{2} & & 0 & \\
& \ddots & & \\
& & w_{r} & & \\
& & 0 & \\
& & & \ddots & \\
& & & & 0
\end{array}\right] .
$$

In the practical calculation, the eigenvalues with small values are truncated to suppress noise amplification. Thus the ratio of $w_{1} / w_{r}$ is treated as the control parameter of the retrieval process. Pseudoinverse matrix $\mathbf{H}^{+}$is obtained as follows,

$$
\mathbf{H}^{+}=\mathbf{V} \mathbf{W}^{+} \mathbf{U}^{T}
$$

where

$$
\mathbf{W}^{+}=\left[\begin{array}{cccccc}
w_{1}{ }^{-1} & & & & \\
& w_{2}^{-1} & & 0 & \\
& & \ddots & & \\
& & w_{r}^{-1} & & \\
& & & 0 & \\
& & & & \ddots & \\
& & & & & 0
\end{array}\right] .
$$

Consequently, the object image is retrieved by the following equation:

$$
\mathbf{f}=\mathbf{H}^{+} \mathbf{g}
$$

For the two-dimensional system, vectors $\mathbf{f}$ and $\mathbf{g}$ and matrix $\mathbf{H}$ become matrices and a tensor. The procedure is the same as for the one-dimensional case described above.

To verify the characteristics of the image-retrieval process, a computer simulation was executed. As configuration parameters, $\mu=10 \times 10, v=22 \times 22$, $a=660 \mu \mathrm{m}$, and $b=45 \mathrm{~mm}$ were assumed. For the system matrix, $\mathbf{H}_{1}$ was calculated from the parameter and $\mathbf{H}_{2}$ was determined experimentally. The width of the point spread was five elements, and the distribution was set to be proportional to the observed distribution. Figure 11 shows an example of the image retrieved by the backprojection method. The reconstructed image is composed of $220 \times 220$ pixels. As one can see from the result, one can obtain a high-resolution image by comparing the origi- 


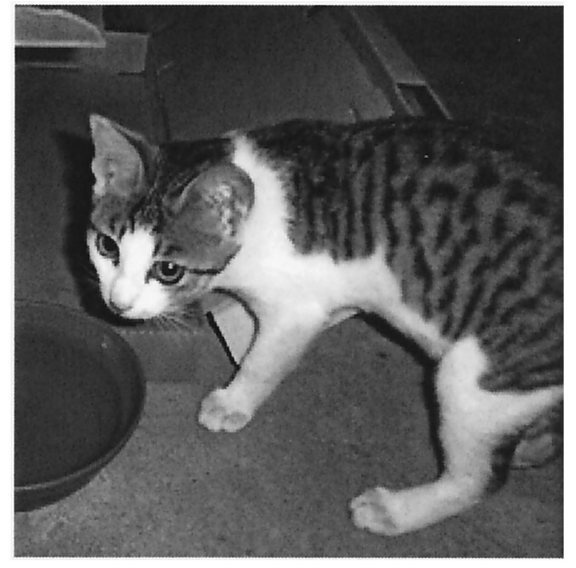

(a)

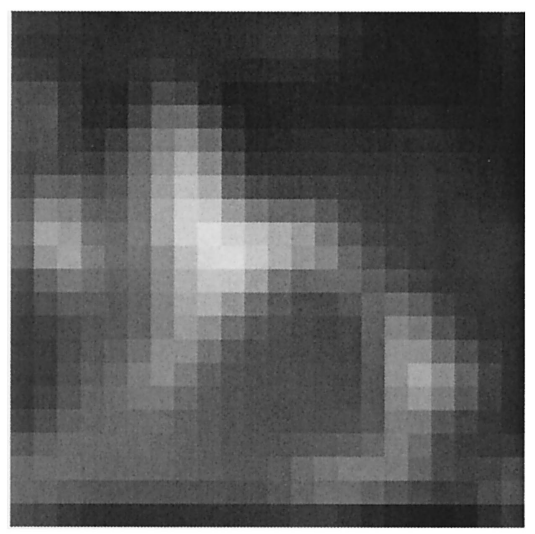

(c)

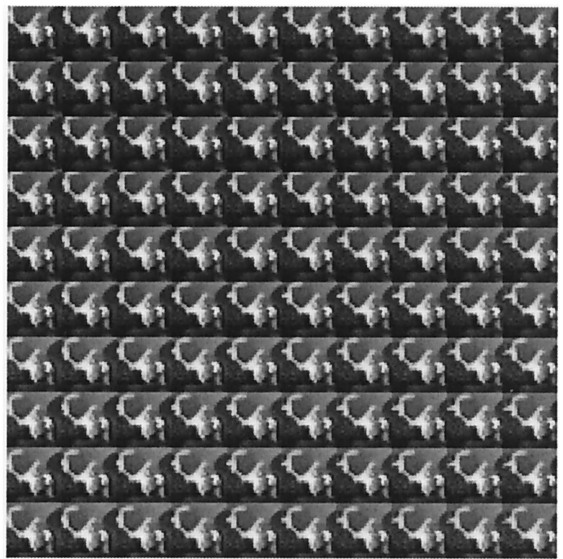

(b)

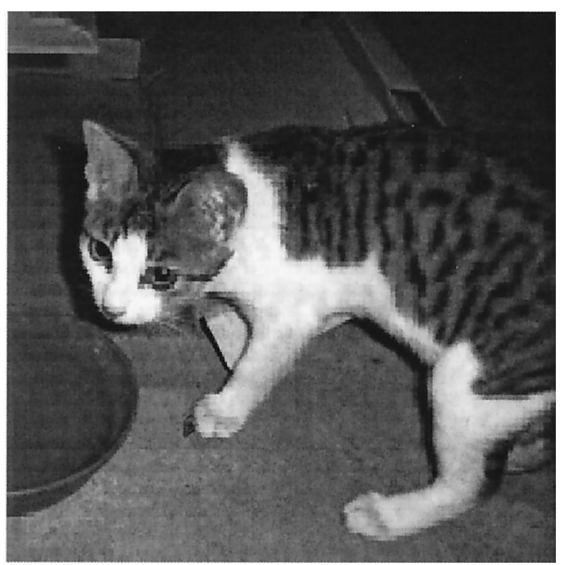

(d)

Fig. 11. Simulation of image retrieval by the backprojection method: retrieved image.

nal image with the image by the image-sampling method.

The backprojection method was applied to the signals captured by the experimental TOMBO system. The object was $10 \mathrm{~mm} \times 10 \mathrm{~mm}$; the distance between the object and the lens, $a$, was $45 \mathrm{~mm}$, and that between the lens and the photodetector, $b$, was 660 $\mu \mathrm{m}$. In terms of the TOMBO system parameters, $\mu=10 \times 10$ and $v=22 \times 22$. Although the experimental TOMBO system has $32 \times 25$ units, we used only $10 \times 10$ to compare the simulation. Because the native $v$ of the experimental system is $22.7 \times$ 22.7 , the detected signals were cast into the form of $22 \times 22$ data by interpolation. Figure 12 shows the image retrieved by the experimental TOMBO system. The ratio of the maximum to the minimum eigenvalues was set as 7 for this case. The image quality was not good, but the edge components of the object were successfully retrieved in comparison with the image observed by a single unit.

\section{Discussion}

In the image retrieval experiment the backprojection method performs better than the sampling method. However, the quality of the image retrieved from the experimental data is not sufficient when it is compared with that retrieved by the simulation. Possible sources of the degradation are aberration and diffraction of the microlenses, residual signal cross talk between the units, misalignment of the components, and signal distortion caused by the analog interface circuits of the CCD camera. The image quality can be improved by solution of these problems one by one.

One of the notable features of the TOMBO architecture is its easy construction by means of stacking the microlens array, the signal separation layer, and the photodetector array. Because no specific device is required for this architecture, large productivity is expected. Relative to the conventional imaging sys- 


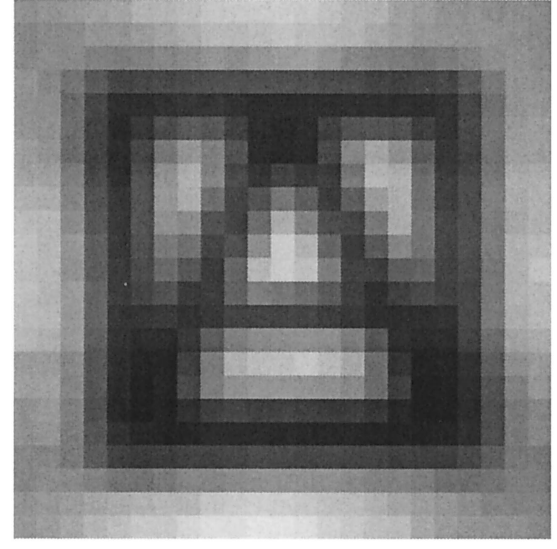

(a)

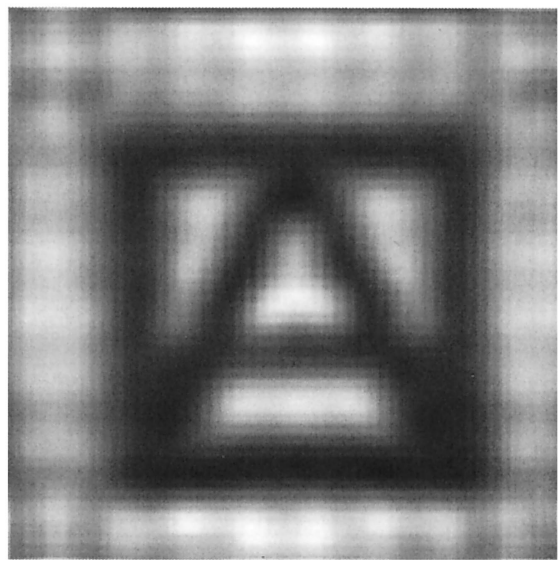

(b)

Fig. 12. Image retrieval by the backprojection method with multiple images captured by the experimental TOMBO system: (a) image of a unit and (b) retrieved image.

tem with single-eye optics, some degree of signal degradation in the TOMBO system is unavoidable owing to the extra signal processing required by the latter. Its applicability to a thin configuration, however, can compensate for the disadvantage. The TOMBO system is an instance of an optoelectronic hybrid's processing systems in which cooperative operation of optics and electronics is a unique feature in the system's configuration.

\section{Summary}

A compact image-capturing system called TOMBO has been presented. A compound-eye imaging system and postprocessing are employed for configuration of a thin system. For image retrieval, two kinds of processing method were considered. Experimental results verify the principle of the proposed method and show the potential capability of the TOMBO architecture to compact an imaging system.

This research was supported by the Development of Basic Tera Optical Information Technologies, Osaka Prefecture Collaboration of Regional Entities for the Advancement of Technological Excellence, Japan Science and Technology Corporation.

\section{References}

1. S. Ogata, J. Ishida, and T. Sasano, "Optical sensor array in an artificial compound eye," Opt. Eng. 33, 3649-3655 (1994).

2. J. S. Sanders and C. E. Halford, "Design and analysis of apposition compound eye optical sensors," Opt. Eng. 34, 222-235 (1995).

3. K. Hamanaka and H. Koshi, "An artificial compound eye using a microlens array and its application to scale invariant processing," Opt. Rev. 3, 264-268 (1996).

4. G. A. Horridge, "Apposition eyes of large diurnal insects as organs adapted to seeing," Proc. R. Soc. London 207, 287-309 (1980).

5. E. Hechit, Optics, 3rd ed. (Addison-Wesley, Reading, Mass., 1998), Chap. 3.

6. K. Yamada, J. Tanida, W. Yu, S. Miyatake, K. Ishida, D. Miyazaki, and Y. Ichioka, "Fabrication of diffractive microlens array for opto-electronic hybrid information system," in Proceedings of Diffractive Optics '99, Vol. 22 of European Optical Society Topical Meetings Digest Series (European Optical Society, Orsay, France, 1999), pp. 52-53.

7. J. Tanida, T. Kumagai, K. Yamada, S. Miyatake, K. Ishida, T. Morimoto, N. Kondou, D. Miyazaki, and Y. Ichioka, "Thin observation module by bound optics (TOMBO): an optoelectronic image capturing system," in Optics in Computing 2000, R. A. Lessard and T. Galstian, eds., Proc. SPIE 4089, 1030-1036 (2000).

8. G. Strang, Linear Algebra and Its Application (Academic, Orlando, Fla., 1976), Chap. 3.

9. T. S. Huang and P. M. Narendra, "Image restoration by singular value decomposition,” Appl. Opt. 14, 2213-2216 (1975). 